\title{
Most Power Reliable Paths Algorithm for Maximizing the Lifetime of Energy Constrained Sensor Networks
}

\author{
Moonseong Kim, Hyunseung Choo ${ }^{\star}$, and Won Kim \\ School of Information and Communication Engineering \\ Sungkyunkwan University, Korea \\ \{moonseong, choo, wonkim\}@skku.edu
}

\begin{abstract}
In wireless sensor networks, maximizing battery life (network lifetime), and thus the number of messages the networks can support (network capacity), is a key issue. In this paper, we propose an algorithm, called Most Power Reliable Paths (MoPoRePa), for online message routing in energy constrained wireless sensor networks. The time complexity of MoPoRePa is significantly lower than the well-known $\max \min z P_{\min }$ algorithm. Moreover, simulation studies show that MoPoRePa about $14.75 \%$ and $8.05 \%$ improvement over the max min $z P_{\min }$ algorithm in terms of network capacity and the network lifetime, respectively.
\end{abstract}

Keywords: Wireless Sensor Networks, Network Capacity, and Network Lifetime.

\section{Introduction}

Wireless sensor networks (WSNs) are composed of low cost sensors. Since low cost sensors typically have short battery life, conserving battery energy is of primary importance [1]. Li et al. 213] proposed an online message routing algorithm, called max min $z P_{\min }$, with the view to maximizing the lifetime of WSNs. To maximize the network lifetime and/or the network capacity, they balance the energy consumed by a route and the minimum residual energy at the nodes along the chosen route. The max min $z P_{\min }$ algorithm selects a unicast path that uses at most $z P_{\min }$ energy, where $z$ is a parameter to the algorithm and $P_{\min }$ is the energy required by the minimum energy unicast path. The algorithm achieves over $80 \%$ of the optimal in terms of network lifetime. However, the time complexity of $\max \min z P_{\min }$ is very high. The reason is that the algorithm involves several invocations of the shortest path computation. The algorithm also requires a lot of time to obtain the $z$ parameter.

In this paper, we propose an algorithm called the Most Power Reliable Paths (MoPoRePa) for online message routing in energy constrained WSNs. Our objectives are as follows. One is to maximize the total number of messages

\footnotetext{
* Corresponding author.
} 
successfully carried by the network (or the network capacity) without making any assumptions on future message arrivals. Another is to increase the network lifetime when the message sequence is not known. We model the lifetime as the earliest time at which communication fails. The MoPoRePa algorithm is very simple, as it requires only one shortest path computation. Further, its performance is better than three previously proposed algorithms in terms of both network capacity and network lifetime. The rest of this paper is organized as follows. Section 2 presents the MoPoRePa algorithm. Section 3 shows the results of simulation studies. Section 4 concludes the paper.

\section{Most Power Reliable Paths Algorithm}

The ad hoc sensor network can be modeled as a directed weighted graph $G=$ $(V, E)$ with node set $V$ and edge (link or arc) set $E$. The energy consumed for transmitting a unit message along link $(u, v) \in E$ is associated with $e_{u v} \geq 0$. Let $P_{u}$ denote the power of node $u \in V$. Formally, we wish to maximize the number of messages in the system subject to the following constraints: 1) the total power used to send all messages from node $u$ does not exceed $P_{u}$ and 2) the number of messages from $u$ to all other nodes is the same as the number of messages from all other nodes to $u$. We assume that our algorithm has knowledge of the topology of the network and the current energy utilization at all nodes in the network. The topology of the network includes all the nodes and links in the network as well as the energy used to transmit a unit message on each link. The network topology can be learned by a node at the time it joins a network.

Using a low energy path frequently leads to energy depletion of the nodes along that path and in the worst case leads to a network partition. We propose an algorithm called Most Power Reliable Paths (MoPoRePa) algorithm. The main idea is to increase the reliability of a path [4. The potential reliable probability that the transmission along the link $(u, v) \in E$ is fault free is $\wp(u, v)=\left(P_{u}^{T}-e_{u v}\right) / P_{u}^{T}$, where $P_{u}^{T}$ is the power of the node $u$ at time $T$. Let $\pi_{s d}$ be a path from source $s$ to destination $d$. The reliability of path $s-d$ is $R\left(\pi_{s d}\right)=\prod_{(u, v) \in \pi_{s d}} \wp(u, v) . \pi_{s d}$ is called a most power reliable $s-d$ path if $R\left(\pi_{s d}\right)$ is maximum among all $s-d$ paths. The link cost function for transmitting a unit message along link $(u, v) \in E$, is defined as $C_{u v}=-\log \wp(u, v)$. Then computing a most power reliable path is equivalent to computing a shortest path using the link cost function $C$. The following summarizes the MoPoRePa algorithm:

\section{MoPoRePa Algorithm}

1. Associate the potential reliable probability with each link.

2. Associate the link cost function with the probability.

3. Find the shortest path from source to destination with the cost. 
Generally, the logarithm function changes the multiplicative routing measure into an additive routing measure as in equation (10).

$$
-\log \prod_{(u, v) \in \pi_{s d}} \wp(u, v)=\sum_{(u, v) \in \pi_{s d}} C_{u v}
$$

Hence, a most power reliable path can be computed in $O(m+n \log n)$ time complexity $(|V|=n,|E|=m)$ using the algorithm of Fredman and Tarjan [5]. In contrast, max min $z P_{\text {min }}$ involves performing as many as $m$ shortest path computations. The running time is at most $O(m(m+n \log n))$. Using binary search, the running time can be reduced to $O(\log m(m+n \log n))[2]$.

\section{Performance Evaluation and Discussion}

For simulation studies of the MoPoRePa algorithm, we generated random networks by modifying the Rodionov and Choo's mechanism [6] to include properties of ad hoc networks. We implemented in $\mathrm{C}$ our method of setting up the paths. We generated 100 different networks with 50 nodes and placed the nodes randomly with density of $0.005 \sim 0.03$ nodes $/ \mathrm{m}^{2}$. We set the transmission radius of each node as $30 \mathrm{~m}$. The power consumption weights for transmitting a message were $e_{u v}=0.001 \cdot d_{u v}^{3}$ [7], where $d$ is a distance, and the initial power of each node is 30 . We generated 1000 messages between all possible pairs of nodes and distributed them evently.

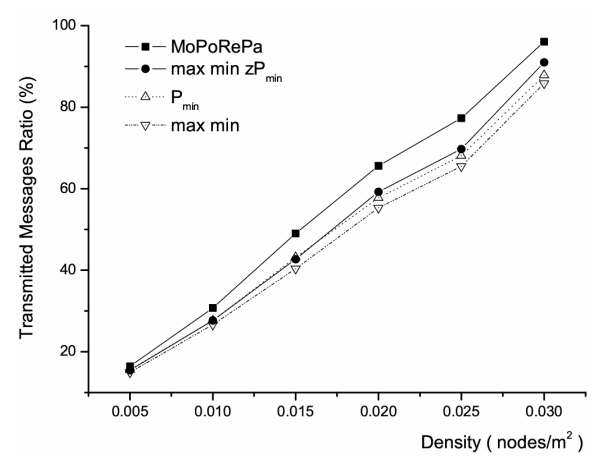

(a) Network capacity

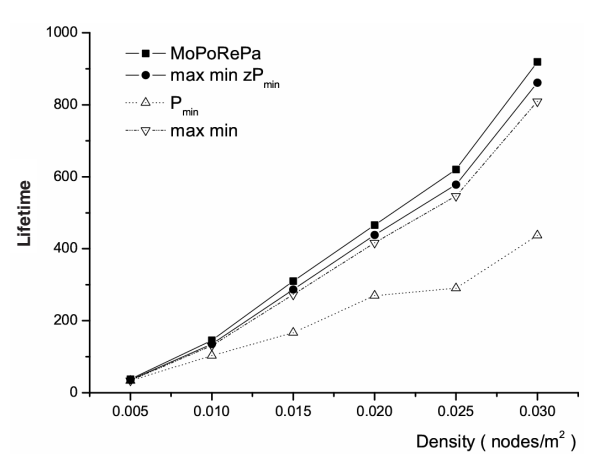

(b) Network lifetime

Fig. 1. Simulation results

We compared the MoPoRePa algorithm with three existing algorithms in terms of the network capacity and the network lifetime. For the $P_{\min }$ algorithm, a message was routed on a path that consumes minimum total energy; i.e., $z$ is 1 in the $\max \min z P_{\min }$ algorithm. For the max min algorithm, a message was routed along a path such that minimum residual energy fraction of nodes on 
that path is the maximum among all paths; i.e., $z$ is $\infty$ in the max $\min z P_{\text {min }}$ algorithm.

Fig. 1(a) shows the simulation results with respect to network capacity. The performance of MoPoRePa algorithm is better than those of the other three algorithms. The improvement is up to about $14.75 \%$ compared to max min $z P_{\text {min }}$. As indicated in Fig. 1(b), the lifetime of MoPoRePa is longer than that of the other three algorithms. MoPoRePa is longer by up to $5.98 \sim 8.05 \%$ compared to max min $z P_{\min }$.

\section{Conclusion}

In this paper, we reported the results of our work on maximizing the lifetime and capacity of energy-constrained wireless sensor networks. In particular, we proposed and validated an online message routing algorithm, called MoPoRePa. The algorithm is worthy because its time complexity is much lower than the bestknown algorithm, the max min $z P_{\text {min }}$ algorithm. Moreover, simulation studies showed that the algorithm is better than the max min $z P_{\text {min }}$, and a few others, with respect to both network lifetime and network capacity.

\section{Acknowledgment}

This research was supported by Ministry of Information and Communication, Korea under ITRC IITA-2006-(C1090-0603-0046).

\section{References}

1. Chang, J.-H., Tassiulas, L.: Energy Conserving Routing in Wireless Ad-hoc Networks. Proceeding of INFOCOM, Vol. 1. IEEE (2000) 22-31

2. Li, Q., Aslam, J., Rus, D.: Online Power-aware Routing in Wireless Ad-hoc Networks. Proceeding of MobiCom. ACM (2001) 97-107

3. Aslam, J., Li, Q., Rus, D.: Three Power-aware Routing Algorithms for Sensor Networks. Wireless Communications and Mobile Computing, Vol. 3. John Wiley \& Sons (2003) 187-208

4. Xue, G.: End-to-End Data Paths: Quickest or Most Reliable?. Communications Letters, Vol. 2, No. 6. IEEE (1998) 156-158

5. Fredman, M. L., Tarjan, R. E.: Fibonacci Heaps and Their Uses in Improved Network Optimization Algorithms. Journal of the ACM, Vol. 34, Issue 3. ACM (1987) 596-615

6. Rodionov, A. S., Choo, H.: On Generating Random Network Structures: Connected Graphs. Lecture Notes in Computer Science, Vol. 3090. Springer-Verlag (2004) 483-491

7. Heinzelman, W. R., Chandrakasan, A., Balakrishnan, H.: Energy-Efficient Communication Protocol for Wireless Microsensor Networks. Proceeding of the 33rd Hawaii International Conference on System Sciences. IEEE (2000) 